\title{
Investigation of mucosal pattern of gastric antrum using magnifying narrow-band imaging in patients with chronic atrophic fundic gastritis
}

\author{
Yasushi Yamasakia,b, Noriya Uedo ${ }^{a}$, Hiromitsu Kanzakia,b, Minoru Kato ${ }^{a}$, Kenta Hamada ${ }^{a}$, Kenji Aoia, \\ Yusuke Tonai ${ }^{a}$, Noriko Matsuura a , Takashi Kanesaka ${ }^{a}$, Takeshi Yamashina ${ }^{a}$, Tomofumi Akasaka ${ }^{a}$, \\ Noboru Hanaoka ${ }^{a}$, Yoji Takeuchia, Koji Higashino ${ }^{a}$, Ryu Ishihara ${ }^{a}$, Yasuhiko Tomitac, Hiroyasu lishia \\ Osaka Medical Center for Cancer and Cardiovascular Diseases, Osaka; Okayama University Graduate School of \\ Medicine, Dentistry, and Pharmaceutical Sciences, Okayama, Japan
}

Abstract

${ }^{a}$ Department of Gastrointestinal Oncology, Osaka Medical Center for Cancer and Cardiovascular Diseases, Osaka (Yasushi Yamasaki, Noriya Uedo, Hiromitsu Kanzaki, Minoru Kato, Kenta Hamada, Kenji Aoi, Yusuke Tonai, Noriko Matsuura, Takashi Kanesaka, Takeshi Yamashina, Tomofumi Akasaka, Noboru Hanaoka, Yoji Takeuchi, Koji Higashino, Ryu Ishihara, Hiroyasu Iishi); 'Department of Gastroenterology and Hepatology, Okayama University Graduate School of Medicine, Dentistry, and Pharmaceutical Sciences, Okayama (Yasushi Yamasaki, Hiromitsu Kanzaki); 'Department of Pathology, Osaka Medical Center for Cancer and Cardiovascular Diseases, Osaka (Yasuhiko Tomita), Japan

Conflict of Interest: None

Correspondence to: Noriya Uedo, MD, Department of Gastrointestinal Oncology, Osaka Medical Center for Cancer and Cardiovascular Diseases, 3-3 Nakamichi 1-chome, Higashinari-ku, Osaka 537-8511, Japan, Tel.: +81 66972 1181, Fax: +81 66981 4067, e-mail: uedou-no@mc.pref.osaka.jp

Received 04 January 2017; accepted 19 February 2017; published online 21 March 2017

DOI: https://doi.org/10.20524/aog.2017.0134
Conclusion White villiform type mucosa is indicative of atrophy and intestinal metaplasia in the gastric antrum. It extends to the whole or central part of the areae gastricae as CAFG becomes more extensive.

Keywords Chronic atrophic fundic gastritis, gastric antrum, intestinal metaplasia, magnifying endoscopy, narrow-band imaging

Ann Gastroenterol 2017; 30 (3): 1-7

\begin{abstract}
Methods Endoscopic images of the greater curvature of the antrum were evaluated in
50 patients with chronic atrophic fundic gastritis (CAFG). The extent of CAFG was evaluated by autofluorescence imaging. The micro-mucosal pattern was evaluated by M-NBI and classified into taken from regions showing different micro-mucosal patterns. Associations among the extent of , micro-mucosal pattern, and histology were examined. Results As the extent of CAFG increased, the proportion of white villiform type mucosa increased,
whereas that of groove type mucosa decreased $(\mathrm{P}=0.022)$. In patients with extensive CAFG, most
of the areae gastricae was composed of the segmental or central type of white villiform type mucosa
$(\mathrm{P}=0.044)$. The white villiform type mucosa had significantly higher grades of atrophy $(\mathrm{P}=0.002)$
and intestinal metaplasia $(\mathrm{P}<0.001)$ than did the groove type mucosa. Results As the extent of CAFG increased, the proportion of white villiform type mucosa increased,
whereas that of groove type mucosa decreased $(\mathrm{P}=0.022)$. In patients with extensive CAFG, most
of the areae gastricae was composed of the segmental or central type of white villiform type mucosa
$(\mathrm{P}=0.044)$. The white villiform type mucosa had significantly higher grades of atrophy $(\mathrm{P}=0.002)$
and intestinal metaplasia $(\mathrm{P}<0.001)$ than did the groove type mucosa. Results As the extent of CAFG increased, the proportion of white villiform type mucosa increased,
whereas that of groove type mucosa decreased $(\mathrm{P}=0.022)$. In patients with extensive CAFG, most
of the areae gastricae was composed of the segmental or central type of white villiform type mucosa
$(\mathrm{P}=0.044)$. The white villiform type mucosa had significantly higher grades of atrophy $(\mathrm{P}=0.002)$
and intestinal metaplasia $(\mathrm{P}<0.001)$ than did the groove type mucosa.

Background Magnifying narrow-band imaging (M-NBI) can reportedly help predict the presence mucosal pattern of the antrum shown by M-NBI differs from that of the corpus. We studied the distribution and histology of the micro-mucosal pattern in the antrum based on magnifying endoscopy. into three types in relation to the areae gastricae: null, central, and segmental types. Biopsies were
\end{abstract}

.




\section{Introduction}

A long-standing Helicobacter pylori (H. pylori) infection causes inflammatory cell infiltration followed by glandular atrophy, intestinal metaplasia in the gastric mucosa, and finally gastric cancer [1]. In particular, the presence of atrophy and intestinal metaplasia is strongly associated with the risk of gastric cancer [2-5]. The current standard for the diagnosis of atrophy and intestinal metaplasia is histological examination of an endoscopic biopsy specimen [6-8]. Basically, biopsies are performed blindly from certain sites of the stomach.

Meanwhile, narrow-band imaging (NBI) endoscopy is reportedly fairly accurate for the diagnosis of atrophy and intestinal metaplasia [9-11]. In our previous study, the micro-mucosal pattern in the gastric corpus was classified as foveolar or groove type using magnifying NBI endoscopy. A foveolar type mucosa in the areae gastricae was less likely to be associated with atrophy and intestinal metaplasia, whereas a groove type mucosa in the intervening part of the areae gastricae was associated with atrophy and intestinal metaplasia [3]. Thus, NBI can be used to predict the presence and distribution of atrophy and intestinal metaplasia in the corpus and may reduce the numbers of biopsy specimens needed and the time required. It is well known that the micro-mucosal patterns of the gastric corpus and antrum are different [12]. In our clinical practice, we have noticed that the micro-mucosal pattern in areas of the gastric antrum affected by intestinal metaplasia was of the white villiform type mucosa, while that in the corpus was of the groove type mucosa. Therefore, in this study, we examined the micromucosal pattern of atrophy and intestinal metaplasia in the gastric antrum using magnifying endoscopy, and compared it with the extent of chronic atrophic fundic gastritis (CAFG).

\section{Patients and methods}

\section{Study design and participants}

This cross-sectional study was conducted at Osaka Medical Center for Cancer and Cardiovascular Diseases from February to December 2010. The study protocol was approved by the ethics committee of our institution and conducted according to the Declaration of Helsinki. Written informed consent was obtained from all patients before enrollment.

We enrolled patients with early-stage non-cardiac gastric cancer who presented to our institution to undergo endoscopic submucosal dissection (ESD). The endoscopic examination was performed before ESD. The patients examined by 2 endoscopists (N.U. and H.K.) were included. The exclusion criteria were a history of $H$. pylori eradication, gastric resection, severe organ failure, and treatment with anticoagulant or nonsteroidal anti-inflammatory medication (NSAIDs) within 7 days of this study.

\section{Endoscopic procedure}

Endoscopic evaluation was performed in a uniform fashion according to a previous study [3]. Esophagogastroduodenoscopy was performed using a video endoscopy system consisting of a light source (CLV-260SL; Olympus Medical Systems, Tokyo, Japan), a processor (CV-260SL; Olympus), a video monitor, and a video endoscope (EVIS-FQ260Z; Olympus) equipped with two charge-coupled devices: one for high-definition white-light imaging and the NBI mode with a zoom function, and the other for the autofluorescence imaging (AFI) mode. First, we evaluated the extent of CAFG in the AFI mode and categorized it into six types according to the Kimura-Takemoto classification $[13,14]$ : AF-C-I, the entire gastric body appeared purple to dark green; AF-C-II, a color border on the lesser curvature was observed at a lower part of the gastric body; AF-C-III, a color border on the lesser curvature was observed at an upper part of the gastric body; AF-O-I, a color border was observed between the lesser curvature and the anterior wall; AF-O-II, a color border was observed between the anterior wall and the greater curvature; and AF-O-III, a color border on the greater curvature was observed proximal to the lower gastric body. CAFG was considered "small" for AF-C-I and AF-C-II, "medium" for AF-C-III and AF-O-I, and "large" for AF-O-II and AF-O-III [3]. Next, $15 \mathrm{~mL}$ of $0.2 \%$ indigo carmine solution was administered onto the gastric mucosa. The micro-mucosal structure of the greater curvature of the antrum was then evaluated by magnifying endoscopy with chromoendoscopy and NBI. We defined the region of interest as a $2 \times 2 \mathrm{~cm}$ area at the greater curvature of the antrum, $3 \mathrm{~cm}$ proximal to the pyloric ring, and took biopsies from the area $[6,15]$. When different micro-mucosal patterns were observed in the region of interest, biopsies were taken one by one from each area. If a single micro-mucosal pattern was observed, one biopsy was taken from the center of the area.

\section{Micro-mucosal pattern}

The type of micro-mucosal pattern was classified into groove and white villiform types $[10,12,16]$. In magnifying chromoendoscopy, groove type mucosa was characterized by ridged mucosal crests divided by continuous grooves; in magnified NBI this type of mucosa appeared as a ridged light brown micro-mucosal structure surrounding dark brown subepithelial capillaries. White villiform type mucosa appeared as whitish patches in non-magnifying NBI and as whitish small villi in which brownish subepithelial capillaries were obscured in magnifying chromoendoscopy and NBI (Fig.1).

\section{Localization of micro-mucosal pattern}

The localization of the white villiform type mucosa was classified into three types in relation to the areae gastricae: null, central, and segmental types (Fig. 2). In the null type, the areae gastricae were composed of only groove type mucosa. 
In the central type, white villiform type mucosa was present in the center of the areae gastricae and was surrounded by groove type mucosa. In the segmental type, the majority of the
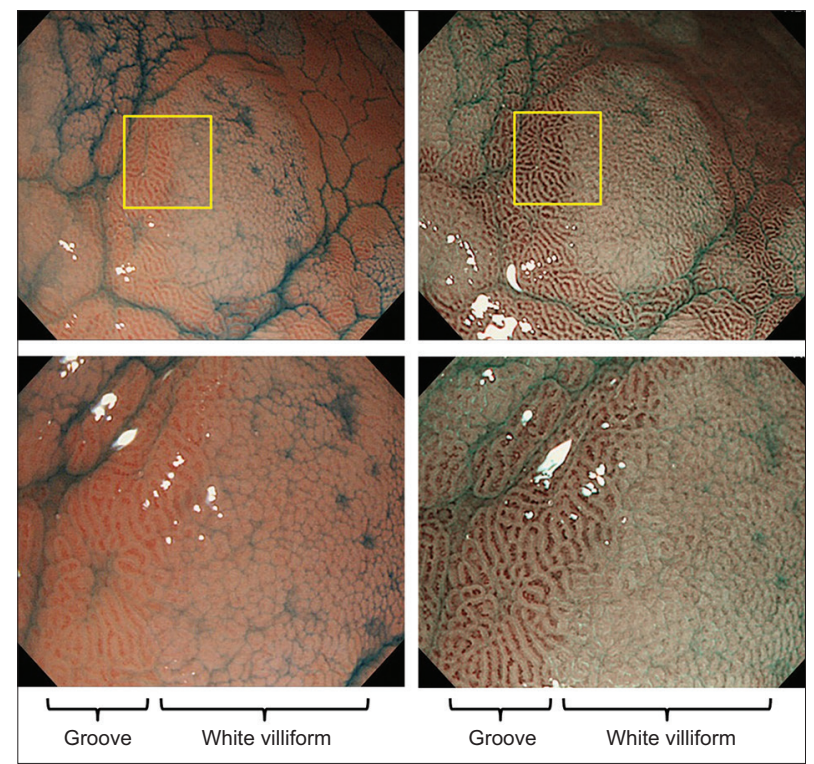

Figure 1 Types of micro-mucosal patterns in chromoendoscopy and narrow-band imaging endoscopy. The micro-mucosal pattern was classified into the groove type or white villiform type areae gastricae was composed of white villiform type mucosa, and none or a minor part was composed of only groove type mucosa.

\section{Proportions of micro-mucosal patterns}

The micro-mucosal pattern in the region of interest was quantitated into four types according to the proportion of groove and white villiform type: white villiform $>80 \%$, almost all areas were composed of white villiform type mucosa; white villiform > groove, white villiform type mucosa occupied 50\% to $80 \%$; white villiform < groove, groove type mucosa occupied $50 \%$ to $80 \%$; and groove $>80 \%$, almost all areas were composed of groove type mucosa.

\section{Histological evaluation}

All biopsy specimens were stained with hematoxylin and eosin. The extent of mononuclear cell infiltration (inflammation), neutrophil infiltration (activity), glandular atrophy (atrophy), and intestinal metaplasia were examined according to the updated Sydney system [6]. All specimens were evaluated by a single experienced pathologist who was blinded to the endoscopic findings.

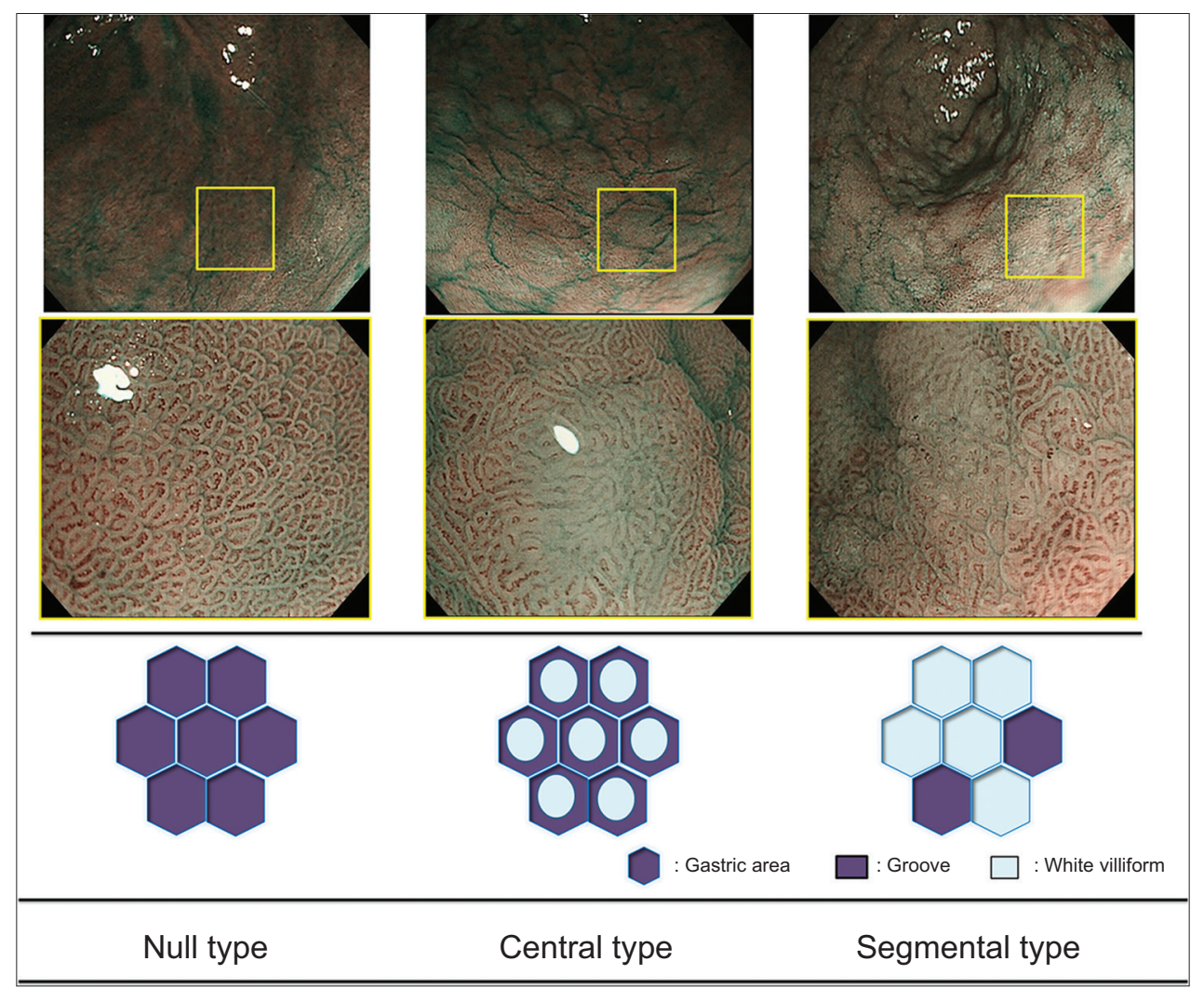

Figure 2 Localization of micro-mucosal structure patterns. The null type was composed of only groove type mucosa. The central type was composed of white villiform mucosa surrounded by groove type mucosa. The segmental type was mainly composed of white villiform mucosa, and the other part was composed of groove type mucosa 


\section{Detection of $\boldsymbol{H}$. pylori infection}

Bacterial culture, serum IgG antibody to $H$. pylori, or histological examination was used to diagnose $H$. pylori infection. At least two of these tests were carried out in all patients. A positive result for any test was diagnosed as "current infection". A negative result for all tests without intestinal metaplasia was diagnosed as "not infected". A negative result for all tests with intestinal metaplasia was diagnosed as "previous or latent infection", because intestinal metaplasia suggests spontaneous eradication or underestimation of $H$. pylori $[17,18]$.

\section{Sample size calculation and statistical analysis}

The sample size was determined according to the feasibility of sample collection and was set at 50 patients in the early part of the study, according to a previous study [3]. All statistical analyses were performed with computer software (JMP version 8; SAS Institute, Cary, NC, USA). The association between the extent of CAFG and the localization or proportion of the micro-mucosal pattern, as well as the grade of histological findings among the different types of micro-mucosal patterns, were analyzed using the chi-square test. A P-value of $<0.05$ was considered statistically significant. The sensitivity, specificity, positive predictive value (PPV) and negative predictive value (NPV) were calculated for detection of any grade of atrophy and intestinal metaplasia compared to no atrophy and intestinal metaplasia.

\section{Results}

From February to December 2010, 190 patients with noncardiac gastric cancer underwent gastric examination before ESD. Of these, 103 patients were examined by other endoscopists who did not participate in this study and 37 did not meet the inclusion criteria (16 had anticoagulant or NSAIDs, 8 had a history of $H$. pylori eradication, 8 had gastric resection and 5 had severe organ failure); thus, 140 patients were excluded from enrollment. A total of 50 patients participated in this study. The patients' characteristics are shown in Table 1 [3].

\section{Association between extent of CAFG and localization of micro-mucosal pattern}

In patients with a small and moderate extent of CAFG, the region of interest consisted almost equally of the null, central, and segmental types. As the extent of CAFG increased, the segmental type increased and the null type decreased. More than $90 \%$ of the patients with widespread CAFG had the segmental type or central type (Fig. 3). There was a significant association between the extent of CAFG and the localization of the micro-mucosal pattern $(\mathrm{P}=0.044)$.
Table 1 Patient characteristics [3]

\begin{tabular}{lc}
\hline Patient characteristics & $\mathrm{N}=50$ \\
\hline Age, years, (range) & $69(45-81)$ \\
Male/female, $\mathrm{n}$ & $44 / 6$ \\
\hline Helicobacter pylori, (\%) & $28(56)$ \\
Current infection & $22(44)$ \\
Previous or latent infection & $0(0)$ \\
Not infected & \\
Extent of atrophic fundic gastritis, (\%) & $7(14)$ \\
Small & $15(30)$ \\
Medium & $28(56)$ \\
Large & \\
\hline Location of tumor & $5(10)$ \\
Upper third & $8(16)$ \\
Middle third & $37(74)$ \\
Lower third & \\
Histology of tumor & $45(90)$ \\
Intestinal type & $5(10)$ \\
\hline Diffuse type &
\end{tabular}

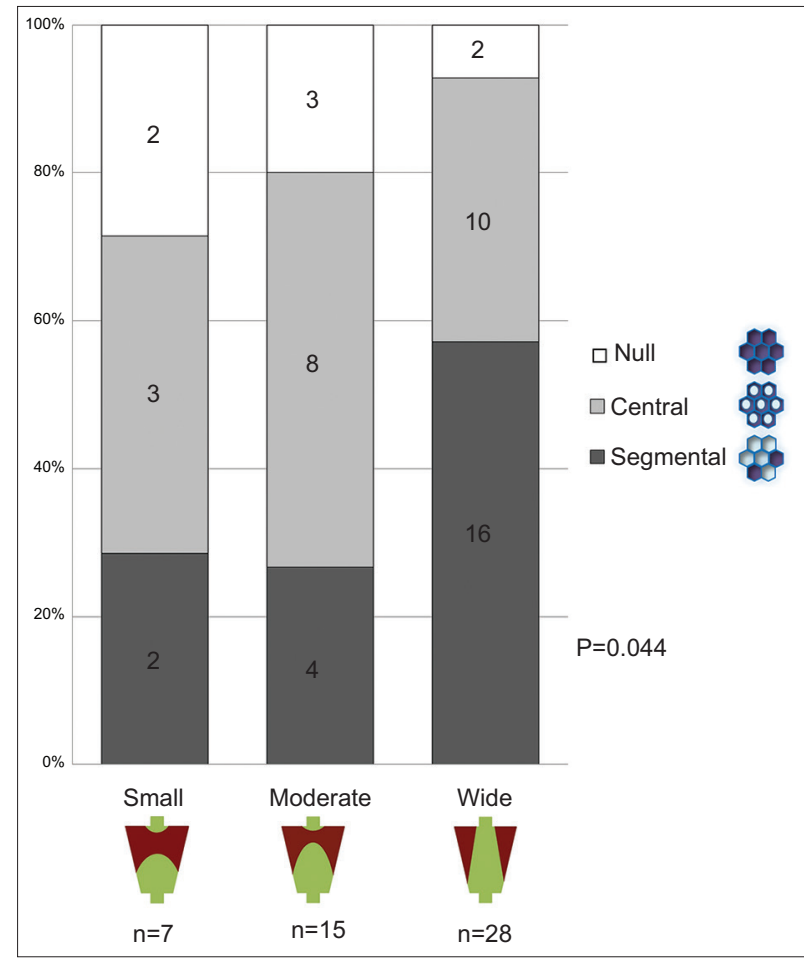

Figure 3 Association between the extent of chronic atrophic fundic gastritis and the localization of the micro-mucosal structure pattern 


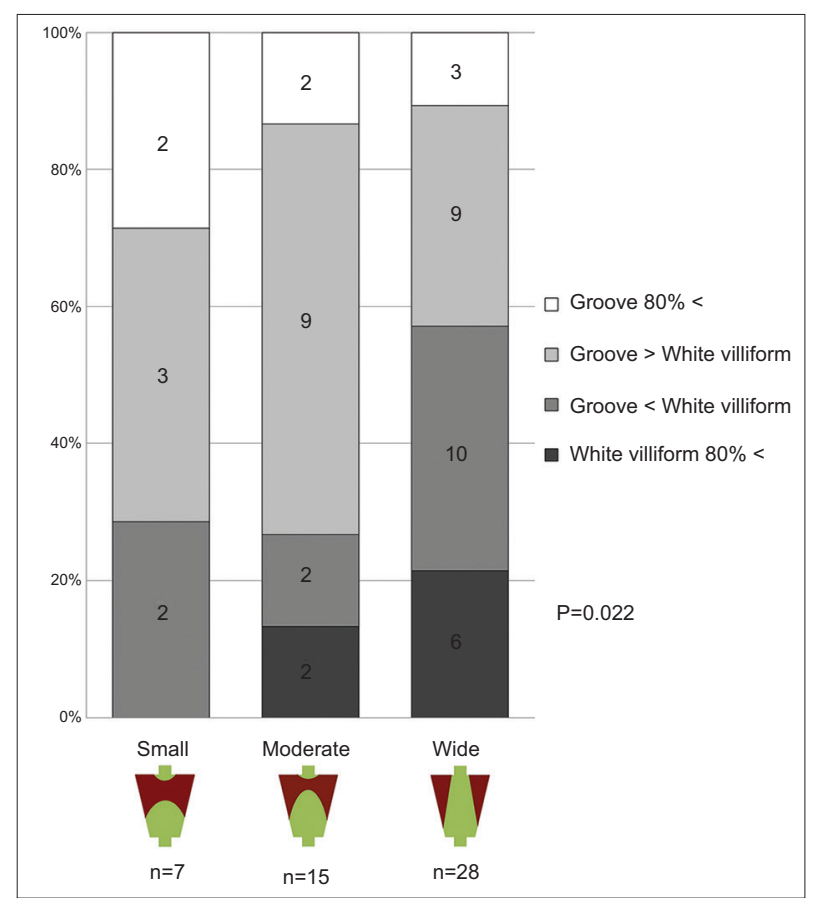

Figure 4 Association between the extent of chronic atrophic fundic gastritis and the proportion of the micro-mucosal structure pattern

\section{Association between extent of CAFG and proportion of micro-mucosal pattern}

The groove type mucosa was dominant in $>70 \%$ of the patients with a small extent of CAFG. As the extent of CAFG increased, the white villiform type mucosa also increased. The white villiform type mucosa was dominant in $57 \%$ of patients with a wide extent of CAFG (Fig. 4). There was a significant association between the extent of CAFG and the proportion of the micro-mucosal pattern $(\mathrm{P}=0.022)$.

\section{Association between histological findings and micro- mucosal pattern}

Among the 50 patients, 45 patients had both groove and white villiform type mucosa. In these patients, two biopsies were taken from mucosa exhibiting each type of micro-mucosal pattern. One biopsy specimen was taken in three patients who had only the groove type mucosa and in two patients with only the white villiform type mucosa. In total, 95 biopsy specimens were taken and evaluated. The white villiform type mucosa had a significantly higher grade of atrophy and intestinal metaplasia than did the groove type mucosa $(\mathrm{P}=0.002$ and $\mathrm{P}<0.001$, respectively). The sensitivity, specificity, PPV, and NPV (95\% confidence interval) of the white villiform type mucosa for histological atrophy were 56\% (44-67\%), 68\% (50-86\%), $83 \%(72-94 \%)$, and 59\% (46-72\%), respectively, and those for intestinal metaplasia were 63\% (52-75\%), 78\% (64-92\%), $85 \%(75-95 \%)$, and $68 \%$ (57-80\%), respectively. There were no significant differences in the grade of neutrophil infiltration or mononuclear cell infiltration between the groove and white villiform type mucosa (Fig. 5).

\section{Discussion}

In this study, we demonstrated that white villiform type mucosa is indicative of atrophy and intestinal metaplasia in the antrum. White villiform type mucosa was localized in the center or the whole segment of the areae gastricae in the antrum. As the extent of CAFG increased, the area with white villiform type mucosa also increased (Fig. 6).

The diagnostic yield of white-light, chromo, and NBI endoscopy for intestinal metaplasia has been discussed previously $[11,19,20]$. In white-light endoscopy, villous mucosa and whitish mucosa were indicative of intestinal metaplasia [19]. In chromo and NBI endoscopy, both the villiform structure and whitish color became more obvious than in white-light endoscopy. In magnifying NBI, a light blue crest [10], white opaque substance (WOS) [21], and marginal turbid band (MTB) [22] were associated with intestinal metaplasia. The WOS and MTB contributed to the whitish color of the intestinal metaplasia, because WOS obscures the brownish subepithelial capillaries, and the MTB clouds the marginal crypt epithelium. Fukuta et al [19] examined the endoscopic appearance of intestinal metaplasia and reported the diagnostic yield using indigo carmine chromoendoscopy. Their endoscopic classification had a sensitivity of $78 \%$, specificity of $58 \%$, PPV of $83 \%$, and NPV of $50 \%$ for intestinal metaplasia in the antrum. Although our results are comparable to theirs, our classifications are simpler and accommodate the progression of the CAFG.

We previously reported on the localization and histology of the micro-mucosal pattern in the corpus in patients with CAFG [3]. The micro-mucosal pattern of the antrum was different from that of the corpus. In the corpus, both normal and non-atrophic/metaplastic mucosa showed a foveola type characterized by mucosa with round or oval foveolae (gastric pits), and the atrophic/metaplastic mucosa showed a groove type characterized by mucosal crests divided by continuous grooves. In the corpus, $>80 \%$ of the groove type mucosa exhibited atrophy and intestinal metaplasia, compared to only $50 \%$ of the groove type mucosa in the antrum. This is why the normal antral mucosa exhibited the groove type; thus, we could not distinguish atrophic/metaplastic mucosa from nonatrophic/metaplastic mucosa with a groove type appearance in the antrum. In the antrum, the white villiform type was more indicative of atrophy and intestinal metaplasia.

In the corpus, groove type mucosa was distributed at the intervening part of the areae gastricae, and the areae gastricae themselves exhibited foveola type mucosa [3]. In the antrum, however, all areae gastricae were divided with narrow furrows, and the intervening part of the areae gastricae was not present; therefore, we classified the localization of the micro-mucosal pattern into null, central, and segmental types, according to the localization of the white villiform type mucosa. In this study, the segmental type corresponded with the wide CAFG in the corpus, suggesting that this is the most advanced distribution status of intestinal metaplasia in the antrum. In one study, the cumulative 5-year incidence of gastric cancer in patients with intestinal metaplasia in only the antrum was $3.3 \%$, comparable with that in patients with intestinal metaplasia in the corpus $(2.7 \%)$ [5]. Thus, the endoscopic diagnosis of intestinal 


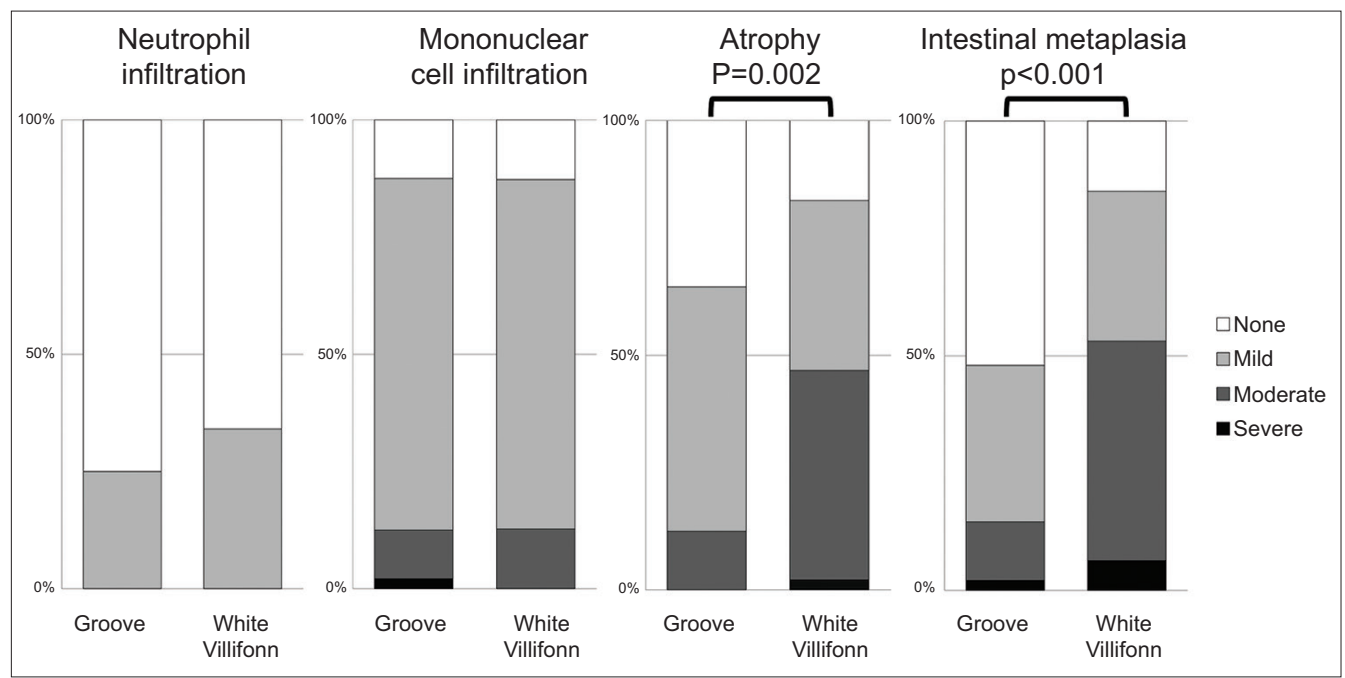

Figure 5 Association between histological findings and the micro-mucosal structure pattern

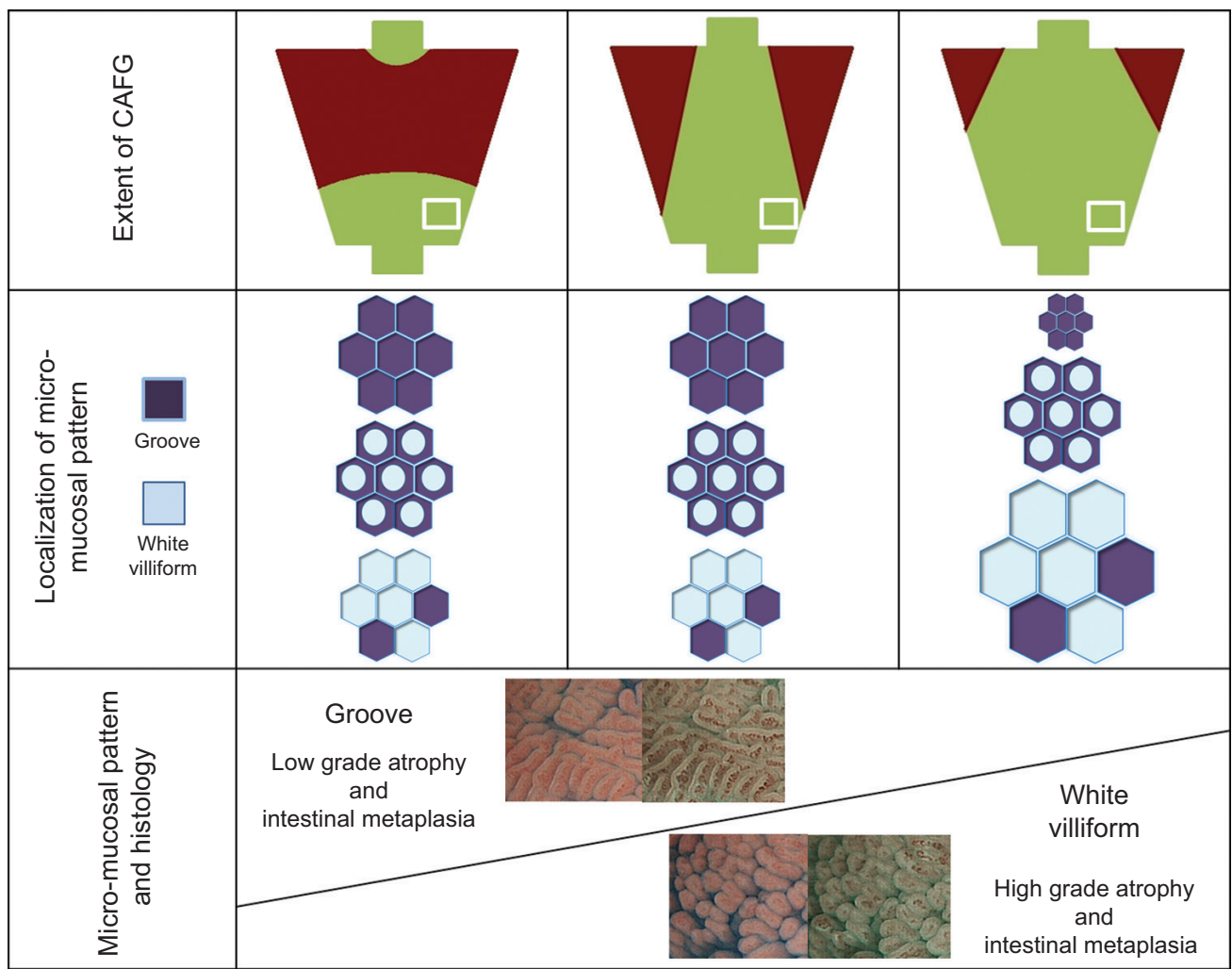

Figure 6 Association among the extent of chronic atrophic fundic gastritis, micro-mucosal structure pattern, and histological findings CAFG, chronic atrophic fundic gastritis

metaplasia in the antrum is important, and a segmental type distribution is likely to indicate a high risk for the development of gastric cancer.

Many studies have evaluated the magnifying NBI findings of chronic gastritis [23], but all studies examined only the association between the endoscopic and histological findings of certain sites. In the present study, however, we clarified the localization and proportion of different micro-mucosal patterns according to the extent of CAFG, suggesting the possibility that endoscopic findings can serve as a marker of the risk of developing gastric cancer.

Although we successfully revealed the association among the extent of CAFG, micro-mucosal patterns and histological findings in the antrum, this study had several limitations. First, it was a single-center analysis with a small sample size, and all patients had early gastric cancer with $H$. pylori-associated gastritis. Thus, whether the concept in this study is applicable to patients without gastric cancer or with other types of chronic 


\section{Summary Box}

\section{What is already known:}

- Intestinal metaplasia is strongly associated with gastric cancer

- Magnifying endoscopy can help to predict the presence of atrophy and intestinal metaplasia

- In the gastric corpus, groove type mucosa exhibits atrophy and intestinal metaplasia

- The micro-mucosal patterns of the corpus and antrum are different

\section{What the new findings are:}

- In the antrum, white villiform type mucosa is indicative of atrophy and intestinal metaplasia

- White villiform type mucosa extends to the whole or central part of the areae gastricae in the antrum as chronic atrophic fundic gastritis becomes more extensive

- The localization and proportion of white villiform mucosa in the antrum might serve as a surrogate marker of the risk of developing gastric cancer

gastritis, such as autoimmune gastritis and lymphocytic gastritis, remains unclear. Second, sampling error should be considered. The groove type mucosa in the central and segmental types was usually small, and the surrounding white villiform type mucosa with atrophy and intestinal metaplasia might therefore have contaminated the biopsy samples.

In conclusion, we clarified the micro-mucosal structure pattern of CAFG in the gastric antrum in relation to the extent of CAFG and histological findings. These findings will improve the endoscopic diagnostic yield in the investigation of the pathogenesis of CAFG.

\section{Acknowledgment}

We express our sincere thanks to the medical staff in our endoscopy unit for their collaboration in this work.

\section{References}

1. Correa P. Chronic gastritis as a cancer precursor. Scand J Gastroenterol Suppl 1984;104:131-136.

2. Uemura N, Okamoto S, Yamamoto S, et al. Helicobacter pylori infection and the development of gastric cancer. $N$ Engl J Med 2001;345:784-789.

3. Kanzaki H, Uedo N, Ishihara R, et al. Comprehensive investigation of areae gastricae pattern in gastric corpus using magnifying narrow band imaging endoscopy in patients with chronic atrophic fundic gastritis. Helicobacter 2012; 17:224-231.

4. Hanaoka N, Uedo N, Shiotani A, et al. Autofluorescence imaging for predicting development of metachronous gastric cancer after Helicobacter pylori eradication. J Gastroenterol Hepatol 2010;25:1844-1849.

5. Shichijo S, Hirata Y, Sakitani K, et al. Distribution of intestinal metaplasia as a predictor of gastric cancer development. J Gastroenterol Hepatol 2015;30:1260-1264.

6. Dixon MF, Genta RM, Yardley JH, Correa P. Classification and grading of gastritis. The updated Sydney System. International Workshop on the Histopathology of Gastritis, Houston 1994. Am J Surg Pathol 1996;20:1161-1181.

7. Rugge M, Meggio A, Pennelli G, et al. Gastritis staging in clinical practice: the OLGA staging system. Gut 2007;56:631-636.

8. Capelle LG, de Vries AC, Haringsma J, et al. The staging of gastritis with the OLGA system by using intestinal metaplasia as an accurate alternative for atrophic gastritis. Gastrointest Endosc 2010;71:1150-1158.

9. Yagi K, Honda H, Yang JM, Nakagawa S. Magnifying endoscopy in gastritis of the corpus. Endoscopy 2005;37:660-666.

10. Uedo N, Ishihara R, Iishi $\mathrm{H}$, et al. A new method of diagnosing gastric intestinal metaplasia: narrow-band imaging with magnifying endoscopy. Endoscopy 2006;38:819-824.

11. Saka A, Yagi K, Nimura S. OLGA- and OLGIM-based staging of gastritis using narrow-band imaging magnifying endoscopy. Dig Endosc 2015;27:734-741.

12. Bansal A, Ulusarac O, Mathur S, Sharma P. Correlation between narrow band imaging and nonneoplastic gastric pathology: a pilot feasibility trial. Gastrointest Endosc 2008;67:210-216.

13. Kimura K, Takemoto T. An endoscopic recognition of the atrophic border and its significance in chronic gastritis. Endoscopy 1969;3:87-97.

14. Inoue $\mathrm{T}$, Uedo $\mathrm{N}$, Ishihara $\mathrm{R}$, et al. Autofluorescence imaging videoendoscopy in the diagnosis of chronic atrophic fundal gastritis. J Gastroenterol 2010;45:45-51.

15. Kimura K. Chronological transition of the fundic-pyloric border determined by stepwise biopsy of the lesser and greater curvatures of the stomach. Gastroenterology 1972;63:584-592.

16. Sakaki N, Iida Y, Saito M, et al. New magnifying endoscopic classification of the fine gastric mucosal pattern. Gastroenterol Endosc 1980;22:377-383. [Japanese with English abstract]

17. Testoni PA, Bonassi U, Bagnolo F, Colombo E, Scelsi R. In diffuse atrophic gastritis, routine histology underestimates Helicobacter pylori infection. J Clin Gastroenterol 2002;35:234-239.

18. Kang HY, Kim N, Park YS, et al. Progression of atrophic gastritis and intestinal metaplasia drives Helicobacter pylori out of the gastric mucosa. Dig Dis Sci 2006;51:2310-2315.

19. Fukuta N, Ida K, Kato T, et al; Study Group for Investigating Endoscopic Diagnosis of Gastric Intestinal Metaplasia. Endoscopic diagnosis of gastric intestinal metaplasia: a prospective multicenter study. Dig Endosc 2013;25:526-534.

20. Kaminishi M, Yamaguchi H, Nomura S, et al. Endoscopic classification of chronic gastritis based on a pilot study by the research society for gastritis. Dig Endosc 2002;14:138-151.

21. Yao K, Iwashita A, Nambu M, et al. Nature of white opaque substance in gastric epithelial neoplasia as visualized by magnifying endoscopy with narrow-band imaging. Dig Endosc 2012;24:419-425.

22. An JK, Song GA, Kim GH, et al. Marginal turbid band and light blue crest, signs observed in magnifying narrow-band imaging endoscopy, are indicative of gastric intestinal metaplasia. BMC Gastroenterol 2012;12:169.

23. Kawamura M, Abe S, Oikawa $\mathrm{K}$, et al. Topographic differences in gastric micromucosal patterns observed by magnifying endoscopy with narrow band imaging. J Gastroenterol Hepatol 2011;26:477-483. 